\title{
Results from e-EVN observations of GRS 1915+105
}

A. Rushton, ${ }^{* 1}$ R. E. Spencer, ${ }^{1}$ M. Strong, ${ }^{1}$ R. M. Campbell, ${ }^{2}$ S. Casey, ${ }^{1}$ R. P. Fender,,${ }^{3,4}$ M. A. Garrett, ${ }^{2}$ J. C. A. Miller-Jones, ${ }^{4}$ G. G. Pooley, ${ }^{5}$ C. Reynolds, ${ }^{2}$ A. Szomoru, ${ }^{2}$ V. Tudose $^{4,6}$ and Z. Paragi ${ }^{2}$

${ }^{1}$ The University of Manchester, Jodrell Bank Observatory, Cheshire SK11 9DA.

${ }^{2}$ Joint Institute for VLBI in Europe, Postbus 2, 7990 A A Dwingeloo, The Netherlands.

${ }^{3}$ School of Physics and Astronomy, University of Southampton, Highfield, SO17 1BJ

Southampton, UK.

4 “Anton Pannekoek” Astronomical Institute, University of Amsterdam, Kruislaan 403, 1098 SJ Amsterdam, The Netherlands.

${ }^{5}$ University of Cambridge, Mullard Radio Astronomy Observatory, J. J. Thomson Avenue, CB3 OHE Cambridge, UK.

${ }^{6}$ Astronomical Institute of the Romanian Academy, Cutitul de Argint, 5 RO-040557 Bucharest, Romania

\begin{abstract}
We present results from the first successful open call e-VLBI science run, observing the X-ray binary GRS 1915+105. e-VLBI science allows the rapid production of VLBI radio maps, within hours of an observation rather than weeks. A total of 6 telescopes observing at $5 \mathrm{GHz}$ across the European VLBI Network (EVN) were correlated in real time at the Joint Institute for VLBI in Europe (JIVE). Throughout this, GRS 1915+105 was observed for a total of 5.5 hours, producing $2.8 \mathrm{~GB}$ of correlated visibility data. The peak brightness was $10.2 \mathrm{mJy}^{-1}$ beam $^{-1}$, with a total integrated radio flux of $11.1 \mathrm{mJy}$.
\end{abstract}

The 8th European VLBI Network Symposium

September 26-29, 2006

Toruń, Poland

*arushton@jb.man.ac.uk 

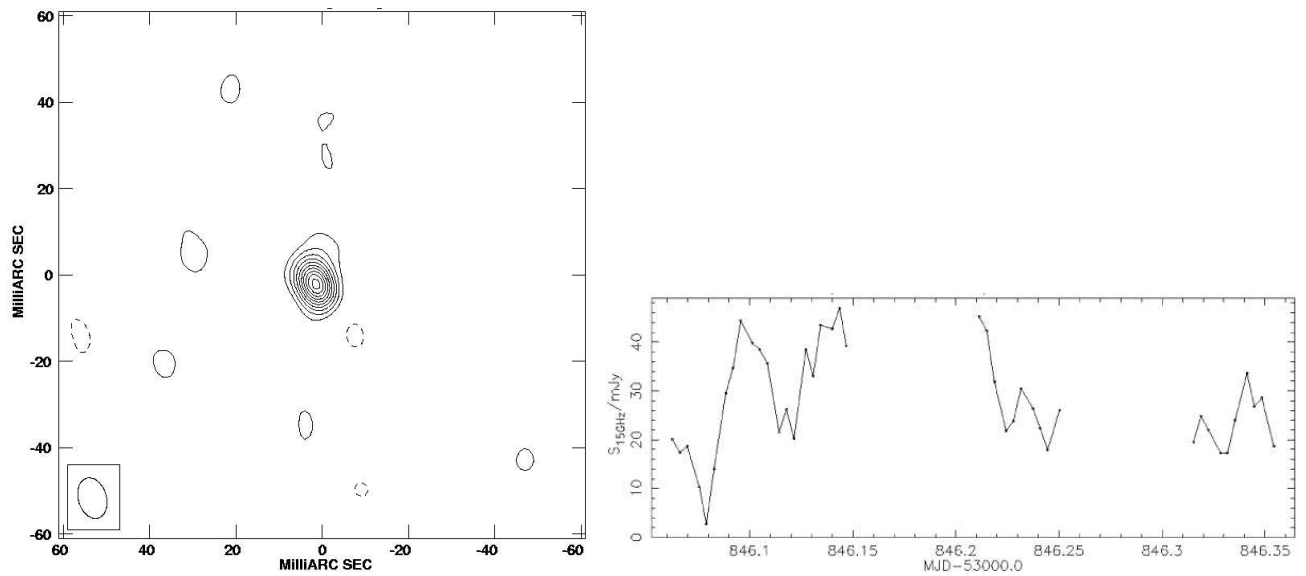

Figure 1: (left) e-EVN map of GRS 1915+105 at $5 \mathrm{GHz}$ using 6 telescopes on 21 April 2006. Contour levels are $(-1,1,2,4,6,8,10) \times 1 \mathrm{mJy}_{\text {beam }}{ }^{-1}$, with an rms of $0.3 \mathrm{mJy}$. (right) Ryle Radio Telescope $15-\mathrm{GHz}$ flux monitoring of GRS 1915+105, carried out at 2006 April 21 01:27 - 08:32 UT.

The use of the Internet for VLBI data transfer offers a number of advantages over conventional recorded VLBI, including improved reliability due to real-time operation and the possibility of a rapid response to new and transient phenomena. Decisions on follow-up observations can be made immediately after the observation rather than delayed by potentially weeks due to problems in shipment of tapes/discs to the correlator. One aim of the project was also to develop a strategy for rapid response (ToO) e-VLBI observations for when this technique is more mature.

In this e-VLBI experiment, the data were transferred from the telescope to the correlator using Mark 5A disk-based VLBI data systems. These units have been fitted with 1-Gbps Network Interface Cards which allow the units to transfer the telescope data to the correlator over the Internet and private optical networks at rates exceeding $100 \mathrm{Mbps}$. Production Internet connections for institutions within each participating country are provided and controlled by the local and national network providers. Most of the telescopes connect to the national networks, and then are connected to the GÉANT $2^{1}$ network allowing pan-European multi-gigabit connectivity. Further details on the Internet connections will be presented by Szomoru et al. (in prep.) and Strong et al. (in prep).

Microquasars are ideally suited for study by e-VLBI since they often have flares associated with the ejection of radio emitting clouds in the form of jets. Time-scales of this emission are in the range of hours to days at $\mathrm{cm}$ wavelengths, and decisions about subsequent observations need to be taken quickly.

The X-ray binary GRS $1915+105$ was first discovered in 1992 [1] by the WATCH instrument on the GRANAT satellite. The system comprises a low mass, K-M III star companion and a $14 \pm 4 \mathrm{M}_{\odot}$ black hole [3]. It was the first Galactic source observed to display superluminal motion, and is well known for its rapid X-ray variability and strong variable radio flux. It spends the majority of its time in relative radio-quiescence, with low radio and X-ray brightness, and with a characteristic low/hard state X-ray spectrum. Transitions to the soft state are often accompanied by strong radio flares with the ejection of a high velocity component out to distances of several hundred milliarcsecond or $\sim 10^{4} \mathrm{AU}$; these transitions have been studied by the VLA and

\footnotetext{
${ }^{1}$ see - http://www.geant2.net
} 
MERLIN [[]]. Long-term high-sensitivity VLBI monitoring of motions in the core is necessary to understand how the inner jets relate to the larger-scale ejections. This is not possible without the strategy in place enabling rapid decisions on follow-up VLBI observations.

On 20 - 21 April 2006 the e-EVN observed GRS 1915+105 at $4.994 \mathrm{GHz}$ using phasereferencing for a total time of 5.5 hours. Each station sustained a transfer rate of $128 \mathrm{Mbps}$ across the network. The radio image of GRS 1915+105 on $20-21$ April 2006 is shown in Fig. 1 (left) using a $u-v$ weighting robustness parameter of 0 . The source had a position of R.A. $=19^{\mathrm{h}} 15^{\mathrm{m}}(11.548 \pm 0.001)^{\mathrm{s}}$ and Dec. $=10^{\circ} 56^{\prime}(44.71 \pm 0.01)^{\prime \prime}(\mathrm{J} 2000)$. The detected source had a major and minor axis of $9.8 \times 6.9$ mas, observed with a beam size of $9.6 \times 6.5$ mas. This was deconvolved with the beam which revealed an extended component of $2.7 \times 1.2$ mas at a position angle of $140( \pm 2)^{\circ}$. This is similar to the P.A. of the large-scale jets previously observed [2]. The total integrated radio flux density was $11.1( \pm 0.6) \mathrm{mJy}$.

Fig. 11 (right) shows the Ryle Radio Telescope flux-monitoring data on 21 April 2006 between 01:27 - 08:32 UT. A flare of 40 mJy was detected, which quickly decayed to $\sim 20$ mJy within 4.5 hours. Assuming equipartition and an isotropic expansion of the flare the minimum energy in the magnetic field for a distance of $11 \mathrm{kpc}[2]$ is $2 \times 10^{41} \mathrm{ergs}$. Using the deconvolved size from the image rather than assuming spherical expansion, we find a minimum energy of $1 \times 10^{40} \mathrm{ergs}$, a lower value due to the source being collimated. This is unlike the major flares studied by the VLA and MERLIN [5] where the decay is over several days and the ejecta can be followed for up to 2 months after the flare. The behaviour of the strong flare is consistent with the shock-in-jet model [ [ ]; however the short flares seem to show the characteristic of an expanding source without continuous ejection of relativistic electrons.

The use of e-VLBI enabled us to obtain images within approximately a day of the VLBI run, rather than the many weeks needed for conventional recording based observations. This work clearly shows the ability of the e-EVN to produce high resolution radio maps in real time, hence eliminating the need of tape/disc recording. In the future, e-VLBI transmission rates will keep increasing with network development, yielding higher sensitivities, and longer baselines will be achieved with the addition of more telescopes to the network. Announcements of opportunity with information on applications are made on the e-EVN web site (http://www.evlbi.org/evlbi) currently every $\sim 2$ months. This is a positive step in the development of a more dynamic and flexible network.

\section{References}

[1] Castro-Tirado, A. J., Brandt, S., \& Lund, N. 1992, IAUC., 5590, 2

[2] Fender, R. P. et al., 1999, MNRAS, 304, 865

[3] Greiner, J., Cuby, J. G., \& McCaughrean, M. J. 2001, Nat, 414, 522

[4] Miller-Jones, J. C. A. et al., 2005, MNRAS, 363, 867

[5] Mirabel, I. F., \& Rodriguez, L. F. 1994, Nat, 371, 46 